Oriolus Revista Científica, ISSN: 2215-6100.1 (1). Julio, 2020, pp 47-57

Salazar Agüero

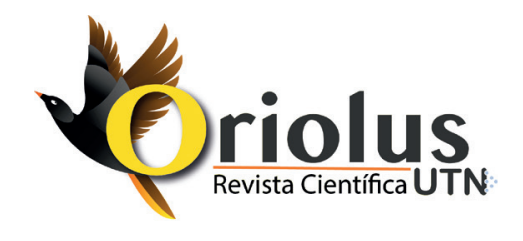

Oriolus Revista Científica

ISSN: 2215-6100

revista.cientifica.oriolus@utn.ac.cr

Universidad Técnica Nacional

\title{
El hidrógeno como Modelo de Descarbonización en Costa Rica
}

\section{Hydrogen as a Decarbonization Model in Costa Rica}

José Heriberto Salazar Agüero

El hidrógeno como Modelo de Descarbonización en Costa Rica

Oriolus Revista Científica, Vol.1, N. ${ }^{0} 1,2020$

Universidad Técnica Nacional

Disponible en: http://revistas.utn.ac.cr/index.php/oriolus

Bibliografía:

Salazar Agüero, Heriberto. "El hidrógeno como Modelo de Descarbonización en Costa Rica". Revista Científica Oriolus 1, N. 1 (2020): 47-57. 
Oriolus Revista Científica, ISSN: 2215-6100.1 (1). Julio, 2020, pp 47-57

Salazar Agüero

\title{
El hidrógeno como Modelo de Descarbonización en Costa Rica
}

\author{
Hydrogen as a Decarbonization Model in Costa Rica
}

José Heriberto Salazar Agüero

Universidad Técnica Nacional, Costa Rica

hsalazar@utn.ac.cr

(iD) https://orcid.org/0000-0002-4431-0882
Recepción: 29 de abril del 2019

Aprobación: 11 de febrero del 2020

\section{Resumen}

Este documento considera los factores positivos y negativos acerca del proceso de descarbonización mediante el uso del hidrógeno, en aras de lograr una economía sostenible y un desarrollo más solidario con el ambiente. Es trascendente reconocer la importancia y los efectos de este tema a largo plazo, sin embargo, se debe revisar cómo se adapta esta tecnología a las áreas productivas en las que es necesario trabajar para alcanzar los objetivos planteados en materia ambiental. Es necesario evidenciar que el tema de descarbonización fortalece y genera una sociedad capaz de convivir sosteniblemente con la naturaleza, además que el uso de hidrógeno como medio de descarbonización conlleva a eliminar las emisiones de gases contaminantes, no obstante, este tópico presenta grandes retos para el desarrollo del país, por el alto costo económico implícito. Asimismo, el desconocimiento de la materia puede provocar que no pase de ser una propuesta, evitando que se concrete en hechos. Es claro que descarbonizar el país es transcendental, pero la propuesta no busca desincentivar el tema ambiental, sino que este estudio invita a incorporar a los todos los sectores a buscar opciones de beneficio en conjunto.

Palabras claves: descarbonización, energía, ambiente, costos, hidrógeno, economía, sector productivo.

\begin{abstract}
This document considers the positive and negative factors about the decarbonization process using hydrogen, in order to achieve a sustainable economy and a more solid development with the environment. It is important to recognize the importance and effects of this issue in the long term, however, it must be verified how this technology adapts to the productive areas in which it is necessary to work to achieve the objectives set in environmental matters. It is necessary to show that the issue of decarbonization strengthens and generates a society capable of living with nature sustainably, in addition that the use of hydrogen as a means of decarbonization leads to eliminating emissions of polluting gases, however, this topic presents great challenges for development of the country, due to the high economic cost implied. Likewise, ignorance of the subject may cause the fleeting observation of the subject, in which it generates that it does not go beyond being a proposal, preventing it from becoming concrete in facts. Decarbonising the country is transcendental, but the proposal does not seek to discourage the environmental issue, but rather that this study invites all sectors to seek benefit options together.
\end{abstract}

Keywords: decarbonization, energy, environment, costs, hydrogen, economy, productive sector.

Esta obra esta bajo una Licencia de Creative Commons Atribución NoComercial-SinDerivar Internacional 
Oriolus Revista Científica, ISSN: 2215-6100.1(1). Julio, 2020, pp 47-57

Salazar Agüero

\section{Introducción}

El proceso de descarbonizar el país para tener una sociedad más sostenible en materia ambiental y económica debe arrojar no solo la admiración de los demás países del orbe, sino que, además, es necesario conocer que al cambiar de bando de movilidad de combustibles con base en carbono a movilidad de energías limpias implica un costo inicial muy elevado para la sociedad. Esta publicación es un estudio que tiene como propósito hacer reflexión sobre la importancia de tener equilibrio en el funcionamiento de las sociedades sostenibles en búsqueda de no ensanchar la brecha social, en el cual hay que concientizar en el tema de la aplicación del hidrógeno, existen ya investigaciones adelantadas por Ad Astra Rocket. Se tiene claro que mejorar los rendimientos de las empresas va ligado al apoyo que el Gobierno ofrezca al sector; no obstante, descarbonizar debe ser una acción que vaya ligada a los objetivos de rentabilización, para que resulte una opción atractiva. El contexto que se construye en este artículo es importante, ya que sensibilizará a la población e incentivará la agilización estatal en la búsqueda de recursos para la implementación de la descarbonización. Sin embargo, surgen dudas sobre si generar y transportar hidrógeno perjudica a los sectores, especialmente, al de transporte. Finalmente, para lograr validar este estudio, se utiliza la metodología DMAIC (Define-Measure-Analize-Improve-Control, por sus siglas en inglés), la cual, a través de estos pasos escalonados, permite lograr el objetivo, puesto que analiza la información recolectada por libros, revistas y noticias.

\section{Materiales y métodos}

A continuación, se presenta el modelo de DMAIC, metodología que busca Definir-Medir-Analizar-Mejorar-Controlar; el cual, se centra en la forma de obtener la información y cómo procesarla, de modo que se genere una discusión técnica en el proceso de esta publicación.

\section{A. Define}

Es necesario definir el problema en cuestión, del cual se está tratando el presente estudio. Se utilizará la herramienta $5 \mathrm{~W}-2 \mathrm{H}$, para definir y explicar el resultado del proceso.

-What: ¿¿ué es la descarbonización?

Según indica Lezama (2018): energías fósiles de bajo contenido de carbono, con alta eficiencia energética" (p. 46), se utiliza el hidrógeno como el elemento relevante en esta técnica.

-Why: ¿Por qué es un reto como país?

Es necesario entender que los gobiernos de turno deben procurar la reactivación económica para reducir el desempleo. La economía es un área en la que el Gobierno busca la descarbonización, pero no cabe duda de que ese proceso debe ser rentable para los diversos sectores y de rápida implementación; de no ser una opción atractiva, se continuará con una economía basada en combustibles fósiles.

-When: ¿Cuándo se debe desarrollar un proyecto de reactivación económica con energías limpias?

Se debe realizar con la mayor brevedad posible, el planeta no espera, la utilización de la energía en el país debe ser renovable e ir mitigando gradualmente el uso de combustibles fósiles. Actualmen-

Esta obra esta bajo una Licencia de Creative Commons Atribución NoComercial-SinDerivar Internacional 
Oriolus Revista Científica, ISSN: 2215-6100.1(1). Julio, 2020, pp 47-57

Salazar Agüero

te, hay un déficit ecológico, debido a que se están consumiendo los recursos naturales de nuevas generaciones.

-Where: ¿¿Dónde es necesario empezar a trabajar sobre estos temas?

Se necesita empezar a laborar en las zonas de mayor incidencia contaminante; la flota vehicular en la gran área metropolitana, según cifras reportadas en el 2008, era de 1200000 vehículos y la tasa de crecimiento anual de $8,5 \%$ (Villegas, 2008).

-Who: ¿Quién es el responsable de empezar a actuar?

Todos los involucrados por el bienestar del país, desde gobiernos centrales, locales, empresas y ciudadanos.

Se ha comenzado por los gobiernos por medio de decretos ejecutivos que incentivan estos proyectos; incluso el Dr. Chang Díaz impulsa el uso de medios de transporte con base en hidrógeno, de ahí que trajo la primera unidad de autobús denominado N' yu' Ti' tal como lo expuso Vargas (2017) en el diario La Nación.

-How: ¿Cómo se debe llevar a cabo la descarbonización?

Se debe empezar con campañas de sensibilización, incentivar ferias informativas en escuelas, colegios y universidades. Además, se necesitan convenios de cooperación del país con naciones pioneras en este campo, que tienen bastante experiencia y cuentan con empresas con una logística muy organizada en este tema. Asimismo, se debe cautivar a la población para que sustituya sus vehículos de combustible por los de energía renovable.

-How Much: ¿Cuánto cuesta sustituir los equipos de producción y de transporte por motores de mayor eficiencia energética?

El costo es sumamente elevado, esta información se ampliará más adelante. A continuación, en la figura 1 se representa la forma de producción de hidrógeno:

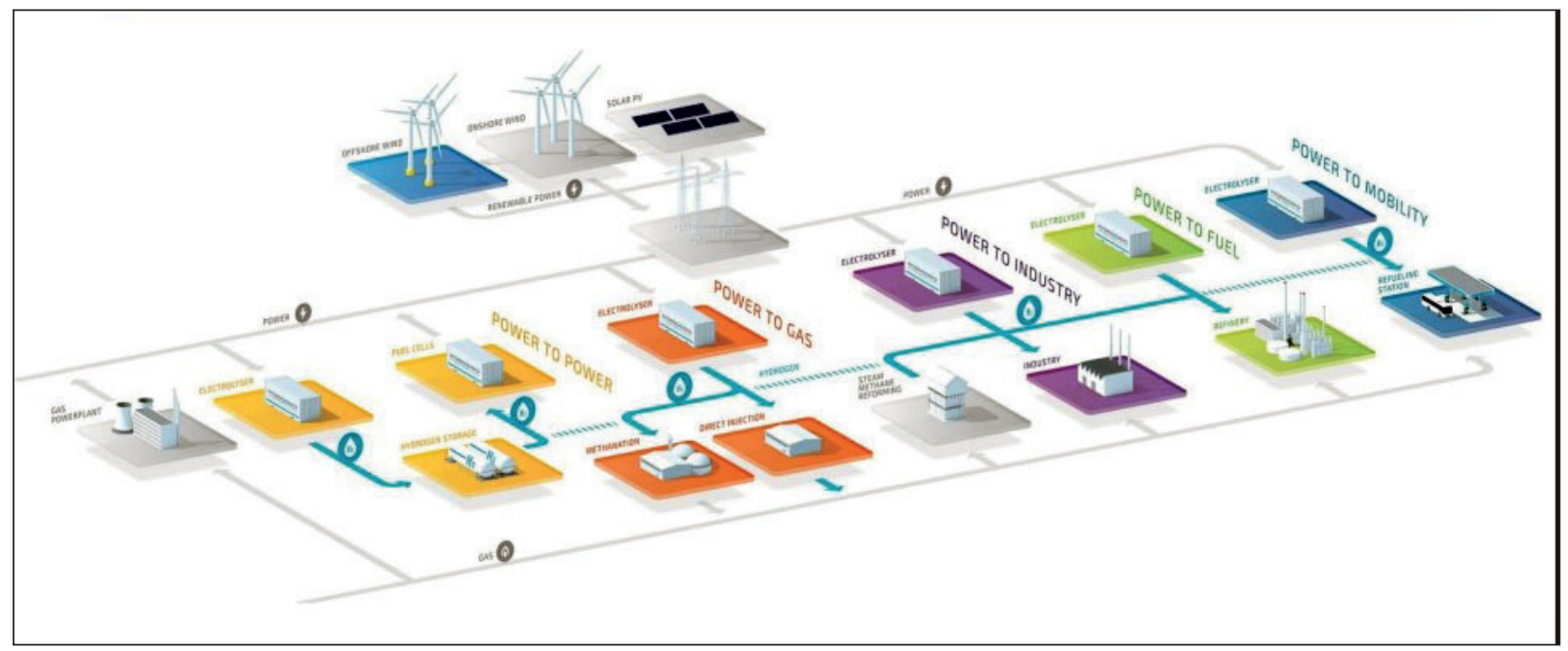

Figura 1. Usos renovables del hidrógeno. Fuente: europeanpowertogas.com, 2020.

Esta obra esta bajo una Licencia de Creative Commons Atribución NoComercial-SinDerivar Internacional 
Oriolus Revista Científica, ISSN: 2215-6100.1(1). Julio, 2020, pp 47-57 Salazar Agüero

\section{B. Measure}

Se establecerá un proceso de medición por medio de la tabla 1 que permita revisar las diferencias entre la utilización de energías fósiles con respecto al uso de energías eficientes, esto enfocado en el sector transporte público (autobuses Higer), debido a que es posible medir el contraste entre motores de hidrógeno con respecto a los de combustibles tradicionales.

Tabla 1. Comparativa de Unidades de Autobuses

\begin{tabular}{|c|c|c|}
\hline Motor & Hidrógeno & Gasolina \\
\hline Costo & $\$ 1000000$ & $\$ 160000$ \\
\hline Autonomía & $340 \mathrm{~km}$ & $440 \mathrm{~km}$ \\
\hline Reabastecimiento & $\$ 190$ (\$5 por 38 gramos) & $\$ 201$ (230 L Diésel) \\
\hline Capacidad & 35 sillas y 70 de pie & 52 sillas y 15 de pie \\
\hline
\end{tabular}

Fuente: elaboración propia, 2020.

Además, otra forma de medición es con los autos convencionales que funcionan como transporte a escala pequeña como los autos, en este caso Hyundai Tucson, como se describe en la tabla 2:

Tabla 2. Comparativa de Autos

\begin{tabular}{|c|c|c|c|}
\hline Motor & Hidrógeno & Gasolina & Eléctrico \\
\hline Costo & $\$ 50000$ & $\$ 32000$ & $\$ 25000$ \\
\hline Autonomía & $400 \mathrm{~km}$ & $180 \mathrm{~km}$ & $238 \mathrm{~km}$ \\
\hline Reabastecimiento & $\$ 25(\$ 5$ por 5 gramos $)$ & $\$ 55(55 \mathrm{~L}$ Gasolina $)$ & $100 \mathrm{Kms} / \$ 2$ \\
\hline Capacidad & 5 asientos & 5 asientos & 5 asientos \\
\hline
\end{tabular}

Fuente: elaboración propia, 2020.

Como se muestra en la tabla 2, existe mayor autonomía y recorrido por parte de un auto que funcione con hidrógeno (a tanque lleno) que uno que se movilice con base en combustible fósil; no obstante, más adelante se observará que los costos de generación de hidrógeno implicarían similitud con el precio que, actualmente, se paga por el combustible diésel y gasolina. 
Oriolus Revista Científica, ISSN: 2215-6100.1(1). Julio, 2020, pp 47-57

Salazar Agüero

\section{Resultados de la Investigación}

\section{Analyze}

En esta etapa es importante realizar las observaciones y planteamientos en función de las decisiones correctas a la hora de invertir; por ejemplo, a una empresa autobusera, que desee convertirse en un modelo carbono neutro por medio de los motores de hidrógeno.

Es claro que, a la hora de comparar precios, se aprecia que el bus o la unidad de hidrógeno cuestan más de seis veces que una unidad equivalente que funcione con gasolina.

Consultando a varios empresarios, coinciden en que con el precio de una unidad de hidrógeno pueden comprar una flotilla nueva de agencia completa y pagar salarios para atender directamente una ruta de forma ininterrumpida.

Ellos comentan que no es únicamente el hecho de que se compran, sino que además pueden gestionar mantenimientos preventivos y correctivos a las unidades teniendo a mano una reserva de repuestos para darle continuidad al servicio y evitar la interrupción parcial de este. No obstante, en la actualidad existe mucho desconocimiento en el ámbito de los equipos y motores de hidrógeno.

Como menciona Otterbach, (2014): "Ias celdas de combustión son muy costosas, debido a que requieren platino como catalizador además que son grandes y frágiles, debido a lo que estas se usan sobre todo en aplicaciones espaciales" (82); esto evidencia que la creación de plantas de producción de hidrógeno es sumamente costosa, lo cual lo vuelve en un reto muy pesado para una economía de baja solvencia. Apoyado en esta misma página, el autor indica que: "Después de la emoción inicial respecto a los coches operados con hidrógeno, varias compañías automotrices han abandonado los prototipos de carros operados con este gas" (82).

Es necesario aclarar que el país no debe hacer inclusión inmediata a este tipo de tecnología para la movilidad. Se debe reconocer que, actualmente existe un proceso de movilidad híbrida que está haciendo su aparición gradual en la industria del transporte; como lo indica Poratti, (2010): "El hidrógeno es demasiado costoso en la actualidad, sin embargo, hay que tomar en cuenta que su producción a gran escala podría bajar los costos en el futuro. A modo de conclusión, bien podríamos sintetizar que en el futuro cercano se impondrán los automóviles híbridos" (102). En el caso del rendimiento respecto a velocidad, frenado entre otros de un motor de combustión de diésel con respecto a un híbrido se muestra la tabla 3:

Tabla 3. Rendimiento Motores Diésel y Gasolina versus Híbrido

\begin{tabular}{|c|l|}
\hline \multicolumn{2}{|c|}{ Rendimiento } \\
\hline Diésel y Gasolina & Híbrido \\
\hline $35-40 \%$ & $90-95 \%$ \\
\hline
\end{tabular}

Fuente:El hidrógeno y las pilas de combustible: Tratamiento y valorización energética de residuos $(2012,1086)$.

Esta obra esta bajo una Licencia de Creative Commons Atribución NoComercial-SinDerivar Internacional 
Oriolus Revista Científica, ISSN: 2215-6100.1(1). Julio, 2020, pp 47-57

Salazar Agüero

Esto es un gran indicador de que el país debe valorar como vía posible, de manera responsable y no realizar impactos sumamente riesgosos en materia económica; está claro que los beneficios ambientales en el uso del hidrógeno están claramente demostrados, sin embargo, no compensan con las necesidades sociales que actualmente aquejan al país.

Además, hay que tomar como referencia lo que indica Castells y Jurado de Gracia, (2012): "la obtención de hidrógeno disponible como combustible a bordo de un vehículo tiene un rendimiento del 15-25 \%" (1087). Lo anterior demuestra que esta tecnología debe ser depurada y puesta al servicio de la sociedad y no precisamente generar un problema como el etanol con RECOPE tal como lo menciona Lara (2019) para el diario La Nación.

\section{Discusión}

El análisis no queda únicamente en el marco comparativo de unidades de buses, sino que el país al declararse carbono neutral se verá obligado a convertirse en productor de hidrógeno para lograr darle soporte a la demanda actual. El jueves 2 de agosto del 2018 en el Colegio Federado de Ingenieros y Arquitectos CFIA se realizó el Simposio: "El hidrógeno en el contexto de fuentes alternas de energía para el transporte", en el cual, el Ing. Carlos Roldán Villalobos expuso y evidenció que, en el escenario más optimista, el hidrógeno producido por electrólisis tendría un costo equivalente al triple del precio actual de la gasolina. "El costo final del hidrógeno, incluyendo el impuesto único a los combustibles, podría bajar en el mejor de los casos a $\not \subset 1.828$ por litro equivalente de gasolina" según informa Roldán (2018) para La Nación. Esto es un hecho preocupante, debido a que no solo se cuestionará la implementación de este tipo de modelo económico sostenible, sino que es claro que no se puede repercutir en aspectos financieros para la población costarricense, ya que perjudicará sin duda a la población con mayores condiciones de vulnerabilidad, lo cual traerá contrastes con el plan de reactivación económica, crucial para Costa Rica.

Por lo tanto, es claro que para que se pueda sustituir en grandes proporciones la utilización de la gasolina por la generación de hidrógeno se necesitarán, aproximadamente, 340000000 kilogramos de hidrógeno al año, para sustituir 1300000000 de litros de gasolina, esta es la cifra consumida que el país consume en el último quinquenio; considerando un artículo publicado por Stafell, Hawkes ym Schmidt denominado "Future cost and performance of water electrolysis: An expert elicitation study" se revelan datos muy importantes a la hora de conocer el procedimiento de la electrolisis, e indica lo siguiente: "plantas electrolíticas necesarias para producir el hidrógeno suficiente para sustituir la gasolina representan una inversión cercana a los \$5 400 millones". A esto, es necesario aunarle, aproximadamente, \$6000 millones para la compra de equipos auxiliares como los dispensadores, compresores, sistemas de seguridad, la logística de almacenamiento y distribución como toda cadena de abastecimiento.

El tema aquí no se detiene, y es necesario exponer tanto los alcances beneficiosos del tema del hidrógeno como las repercusiones; hay que tomar en cuenta que en términos de energía útil $1 \mathrm{~kg}$ de hidrógeno equivale a 3,78 L de combustible. En una entrevista realizada por el diario la Nación en el 2018 al Coordinador de Programa de Energías Limpias del Tecnológico de Costas Rica (TEC) argumentaba que la Organización Energy Information Administration (EIA) del Departamento de Energía de los Estados Unidos, aduce que para el año 2022 el precio de la energía producida por medio de paneles solares significaría el semejante a $\not 34 \mathrm{kWh}$, por lo cual la electricidad solicitada para generar 1 kg de hidrógeno costaría $\not 2.040$ y representaría $\not 540$ por litro de gasolina.

Esta obra esta bajo una Licencia de Creative Commons Atribución NoComercial-SinDerivar Internacional 
Oriolus Revista Científica, ISSN: 2215-6100.1(1). Julio, 2020, pp 47-57

Salazar Agüero

No obstante, para buscar el objetivo de la eficiencia energética para generar hidrógeno con base en energías renovables, esta misma organización muestra que cada planta solar trabajaría únicamente con el 33 \% del período, por lo cual se requeriría aumentar casi tres veces la inversión para generar el hidrógeno solicitado cuando haya luz solar, por lo que en este caso el costo para rentabilizar la producción de hidrógeno alcanzaría a $\not \subset 1.836$ por litro de gasolina y el costo general del hidrógeno, al retener el tributo a los combustibles, lograría los $\not 2.622$ por litro de gasolina, lo que cuadruplicaría el valor del combustible.

A modo de conclusión, la inversión demandada para reemplazar el dispendio de derivados del petróleo en Costa Rica por hidrógeno puede acaparar costos cercanos a $\$ 47.000$ millones de dólares, cifra que puede ser insostenible para el Estado y sus ciudadanos, debido a la condición fiscal en que se encuentra el país; eso equivale prácticamente a dos veces la deuda externa del Gobierno.

\section{Mejorar}

El gran reto de cómo lograr la rentabilidad en un tema que ha generado muchas opiniones respecto al tópico de descarbonización por medio del hidrógeno sigue siendo incógnito y desconocido. Empero, se puede buscar ayuda de países que han mejorado su técnica en los últimos años como Alemania, Rusia o Estados Unidos. Por consiguiente, Se puede reflexionar a partir de un conjunto de preguntas generadoras que buscarán darle nuevas formas de soporte a la descarbonización en el país.

Para ello, es importante utilizar la herramienta SCAMPER. (Sustituir-Combinar-Adaptar-Modificar-Poner en otros usos-Eliminar-Revertir o Arreglar) que permite plantear unas preguntas generadoras que ayudan en esta etapa de la metodología, por lo que se presentan a continuación:

-Sustituir: ¿Qué procedimiento se podría sustituir para la utilización del hidrógeno?

Está claro que, antes de incursionar por cuenta propia, se debe buscar la colaboración de los países pioneros en este modelo energético, de modo que deberían existir convenios para el equipamiento.

-Combinar: ¿Qué otro tipo de movilidad energética eficiente se puede utilizar y combinar para el país?

La parte de electricidad no necesariamente debe ser autónoma, una movilidad eléctrica con baterías puede ser un excelente complemento en comparación con el hidrógeno (con aceptable rentabilidad), ya que podría ser un elemento por considerar, debido a que puede ser autosuficiente y renovable.

-Adaptar: ¿Qué modelo internacional se puede adaptar al país?

Existen países que trabajan este tipo de producción; el Dr. Frankling Chang promueve el modelo que empieza a tener gran auge en Estados Unidos.

-Modificar: ¿Qué elemento modificaría el alto costo en el país para la producción energética por medio del hidrógeno?

Se deberían disminuir los precios de generación en energía con la producción de gran escala de hidrógeno, de modo que no comprometa tanto al cliente a la hora de pagar por kilogramo.

Esta obra esta bajo una Licencia de Creative Commons Atribución NoComercial-SinDerivar Internacional 


\section{Oriolus Revista Científica, ISSN: 2215-6100.1 (1). Julio, 2020, pp 47-57 Salazar Agüero}

-Otros usos: ¿̇Qué otros usos se pueden dar para que diversifique el uso del hidrógeno?

No solo en los motores y en el área de transportes se pueden dar uso al hidrógeno, sino que, en el ámbito industrial incluso, se puede sustituir la parte de movilidad en equipos que requieren combustibles por aplicaciones con hidrógeno, lo cual puede reducir los costos operativos, mayores contrataciones y mejor reactivación de la economía.

-Eliminar: ¿¿Qué factor eliminaría del proceso de electrólisis?

El transporte a largas distancias del hidrógeno, que tiene un alto costo.

-Revertir: ¿¿Ué se puede mejorar respecto a la generación eficiente de energía?

Este país es rico en biodiversidad, por lo tanto, al contar con una consultoría de profesionales muy calificados se puede potenciar la producción de energía con recursos renovables y caminar con más firmeza hacia la ruta del país carbono neutro.

\section{E. Control}

Es claro que mantener el control sobre un proceso implicará mayor desarrollo y regularidad, por lo cual es preferible realizar las correcciones primero e implementar las mejoras conforme a las posibilidades del país, gradualmente, se convierte en carbono neutro. La repetitividad de buenas y eficientes prácticas de generación de energía colaborará con un mayor desarrollo.

La sociedad costarricense está lista para dar ese paso, pero debe haber una práctica sostenible para todos, debe marcar la economía participativa a todos los sectores y no solo mirar el efecto de carbono neutro como una etiqueta internacional.

\section{Conclusiones}

Es importante que el país opte por establecer una economía carbono neutro, que sea sostenible para todas las áreas productivas.

No existe un acceso a este tipo de tecnologías en generación de hidrógeno para la eficiencia energética en Costa Rica.

El proceso de generación de hidrógeno es cuatro veces más costoso que el de combustible convencional.

El costo de los medios de transporte masivo de motores de hidrógeno es seis veces mayor a una unidad convencional, pero de alta tecnología.

La producción de hidrógeno es una necesidad para el país, por la urgencia de mitigar las emisiones de carbono.

Por su alto costo, el hidrógeno parece ser un lujo inconcebible para un país en vías de desarrollo, o al menos valorando la opción en este contexto específico.

Pese a los impulsos gubernamentales y del Dr. Franklin Chang Díaz para que el tema de hidrógeno 
Oriolus Revista Científica, ISSN: 2215-6100.1(1). Julio, 2020, pp 47-57

Salazar Agüero

sea participativo en el país, no existe un estudio de relación costo-beneficio que acople la idea al mercado costarricense.

Según criterios expertos producir hidrógeno en el país es incrementar 200 \% la deuda nacional.

No existe un plan estratégico nacional para impulsar el desarrollo de la descarbonización que simultáneamente reactive la economía.

Otras medidas como los carros eléctricos empiezan a ser una alternativa más factible para el país mejor que el hidrógeno debido a que ya existe comercialización.

\section{Agradecimiento}

A la Universidad Técnica Nacional por su respaldo ante nuestro trabajo académico, abrir sendas de conocimiento para aportar a la sociedad costarricense, fortalecer los vínculos con diversos sectores, además de promover la ejecución de un modelo educativo sostenible para beneficio del cuerpo docente y estudiantil.

\section{Bibliografía}

Castellanos, P. R. Energías y medio ambiente. Ediciones Universidad Salamanca, 2004.

Castells, X. E., \& de Gracia, L. J. El hidrógeno y las pilas de combustible: Tratamiento y valorización energética de residuos. Ediciones Díaz de Santos, 2012.

Castells, X. E. Energías renovables: Energía, Agua, Medioambiente, territorialidad y Sostenbilidad. Ediciones Díaz de Santos, 2012.

Hortal, M. A., \& Barrera, A. L. M. El hidrógeno: fundamento de un futuro equilibrado. Ediciones Díaz de Santos, 2012.

La Nación, Grupo Nación. "Costa Rica cuenta con su primer autobús eléctrico de hidrógeno". 28 de marzo 2019. https://www.nacion.com/tecnologia/innovaciones/costa-rica-cuenta-con-su-primer-autobus-electrico-de-hidrogeno/DBEV45T2JFHQTBSPMWOK2WQTA4/story/

La Nación, Grupo Nación. "Gobierno suspende plan para mezclar etanol con gasolina al menos un año". 10 de enero del 2020. https://www.nacion.com/el-pais/servicios/gobierno-suspende-plan-para-mezclar-etanol-en/LTYVAG4HNZGJNKYWWVTK2GSJYE/story/

La Nación, Grupo Nación. "El hidrógeno en números". 10 de enero del 2020. https://www.nacion. com/opinion/foros/el-hidrogeno-en-numeros/AA322LTHIFH2BL7TNKXVIOQF7A/story/

La Nación, Grupo Nación. "El hidrógeno no es la solución". 28 de marzo del 2019. https://www. nacion.com/opinion/foros/el-hidrogeno-no-es-la-solucion/LKHZFW42PFHFFNXMZ4YQWSQTAE/ story/2 li_source=LI\&li_medium=bottom_article\&li_pl=c 1 4ee 158-cb2b-4532-9221-afbf7f2e34fc\&li_tr=bottom_article

Larepublica.net. "Transportarse 100 kilómetros en Costa Rica en auto es posible con $\not 1.200 " .10$ de enero del 2020. https://www.larepublica.net/noticia/transportarse-100-kilometros-en-costa-rica-en-auto-es-posible-con-1200

Esta obra esta bajo una Licencia de Creative Commons Atribución NoComercial-SinDerivar Internacional 
Oriolus Revista Científica, ISSN: 2215-6100.1(1). Julio, 2020, pp 47-57 Salazar Agüero

Lezama, J. L. Cambio climático, ciudad y gestión ambiental: Los ámbitos nacional e internacional. El Colegio de México AC., 2018.

Llorca Piqué, J. El hidrógeno y nuestro futuro energético. Universitat Politécnica de Catalunya, 2010.

Otterbach, D. H. Energía y Calentamiento Global: ¿̇Cómo asegurar la supervivencia de la humanidad? Grupo Editorial Patria, 2014.

Poratti, G. G. LOS PRÓXIMOS 500 AÑOS ¿ Cómo evolucionaran las casas, computadoras, automóviles, industrias, y robots del futuro? Gustavo Gabriel Poratti, 2010.

Roldán, C. "El hidrógeno en números". La Nación. 28 de marzo del 2019. https://www.nacion.com/ opinion/foros/el-hidrogeno-en-numeros/AA322LTHIFH2BL7TNKXVIOQF7A/story/?li_source=LI\&li_ medium=bottom_article\&li_pl=c 14 ee 158-cb2b-4532-9221-afbf7f2e34fc\&li_tr=bottom_article

Salazar, D. (2019). "Autobús de hidrógeno se pone en marcha en Costa Rica". Semanario Universidad. 28 de marzo del 2019. https://semanariouniversidad.com/pais/autobus-hidrogeno-se-pone-marcha-costa-rica/

Staffell, I., Hawkes, A. and Schmidt, O. Future cost and performance of water electrolysis: An expert elicitation study. ScienceDirect. London: ELSEVIER, 2017.

Villegas, J. "Empeora contaminación del aire en San José". La Nación. 21 de mayo del 2008. Recuperado el 10 mayo de 2019, http://www.nacion.com/In_ee/2008/mayo/21/pais1544915.html 